Association for Information Systems AIS Electronic Library (AISeL)

Wirtschaftsinformatik Proceedings 2001

Wirtschaftsinformatik

September 2001

\title{
Serviceflow Management for Health Provider Networks
}

Ralf Klischewski

Hamburg University, klischewski@informatik.uni-hamburg.de

Ingrid Wetzel

HamburgUniversity, wetzel@informatik.uni-hamburg.de

Follow this and additional works at: http://aisel.aisnet.org/wi2001

\section{Recommended Citation}

Klischewski, Ralf and Wetzel, Ingrid, "Serviceflow Management for Health Provider Networks" (2001). Wirtschaftsinformatik

Proceedings 2001. 14.

http://aisel.aisnet.org/wi2001/14

This material is brought to you by the Wirtschaftsinformatik at AIS Electronic Library (AISeL). It has been accepted for inclusion in Wirtschaftsinformatik Proceedings 2001 by an authorized administrator of AIS Electronic Library (AISeL). For more information, please contact elibrary@aisnet.org. 
In: Buhl, Hans Ulrich, u.a. (Hg.) 2001. Information Age Economy; 5. Internationale Tagung Wirtschaftsinformatik 2001. Heidelberg: Physica-Verlag

ISBN: 3-7908-1427-X

(C) Physica-Verlag Heidelberg 2001 


\title{
Serviceflow Management for Health Provider Networks
}

\author{
Ralf Klischewski, Ingrid Wetzel \\ Hamburg University
}

\begin{abstract}
Exploding expenditure in healthcare has led to new reimbursement regulations forcing healthcare providers to conduct their organizations as business concerns. In order to be competitive providers have started to build networks that allow the delivery of interrelated health services in a well-adjusted and uniform manner. However, besides strategic agreement, successful networking requires the support of information systems in order to deliver efficient day to day service.

With serviceflow management we provide a general concept that answers these needs. Based on the exchange of XML-representations of process knowledge and data between service providers, serviceflow management supports organizations in jointly delivering services that cross provider boundaries. Furthermore, it enables flexible handling of processes, which is indispensable in healthcare. Based on a healthcare example, we explore the possibilities of serviceflow management and present a web-based prototype realized on the basis of our generic four-layered architecture.
\end{abstract}

Keywords: Cross-Organizational Service Processes, Serviceflow Management, EBusiness, E-Health

\section{Introduction}

Today, healthcare providers of all kinds face extreme pressure. Owing to demographic reasons and the availability of better medical facilities and therapy forms, expenses in the healthcare sector are exploding. Consequently, cost reduction is of prime importance. As a response, drastic changes in reimbursement procedures cause healthcare providers to act as firms with a business orientation. The ensuing competition compels healthcare providers either to specialize or merge or both. A promising alternative is to build networks that enable providers to act in a corporate way vis-a-vis business partners (insurance companies or suppliers) as well as clients (patients). This situation only mirrors developments that already exist in the US, such as HMOs (health management organizations). 
The question is whether new web-based technology can improve the situation. Out of the great number of possibilities in online health provision [Ripp00;Rodr00; Tam*00] we are interested in the impact and design of e-business at the joint between networks and patients. Our intention is to support health providers in both a cost effective provision of cross-organizational service processes and a substantial improvement in their quality.

Our work is based on previous experience in healthcare projects [Kra*96; KrWe00; Wetz01]. Like many others, we consider the difficulties in implementing information systems into hospitals as being rooted in the specifics of the domain, such as situated cooperation of high complexity [WoKa97], work "on" human beings [Str*97], hospitals as representatives of special organizational types [Mint79] and relatively low investments and IT knowledge compared to business organizations of similar complexity [Ande97]. We assume these factors will similarly affect efforts in improving cross-organizational processes.

The serviceflow management (SFM) concept presented in this article claims to be suitable in this context. It was developed to support interrelated services and to enable flexibility according to customer needs. Cost effectiveness is achieved by routinization based on standard process pattern. Furthermore, it enables connectivity between heterogeneously IT-equipped network partners by using XMLbased representations for exchanging information between providers.

Our line of argument is as follows. Section 2 introduces a typical cross-provider healthcare service process with existing problems and conflicting perspectives. We conclude with specific requirements for online support. Section 3 presents the general concept of SFM, its aims and a brief architectural description. Section 4 discusses a suitable infrastructure and presents a prototype for the given example. A summary highlights the main possibilities of SFM in healthcare provider networks and how it could contributes to new e-health scenarios.

\section{Cross-Organizational Healthcare Service Processes}

Apart from cost reduction, the nature of health service itself gives grounds for networking. In the case of a more severe disease, a close cooperation among specialized healthcare providers is required in order to enable a comprehensive diagnostic, curative and care process. 


\subsection{The Case: Preparation, Performance and Aftercare of an Inpatient Surgical Operation}

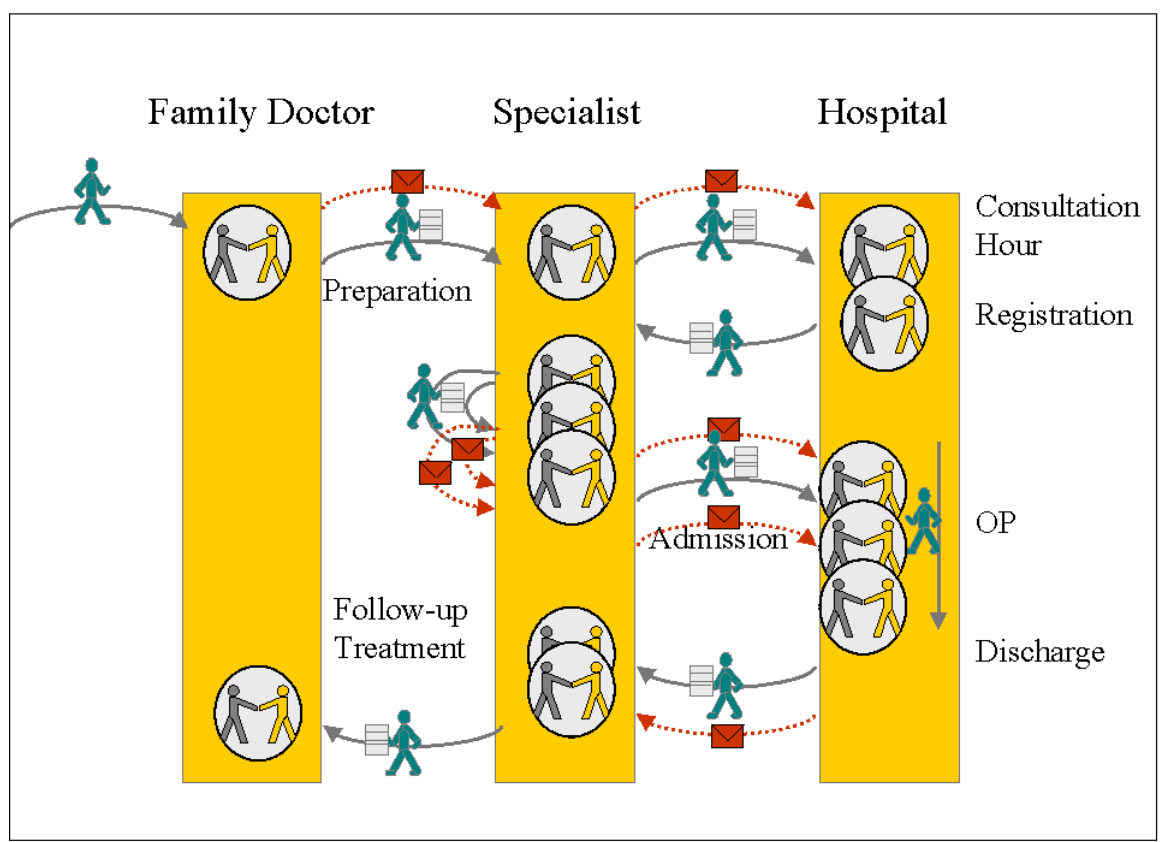

Figure 1: Service Example: Preparation, Performance and Aftercare of an Operation.

Based on our analysis in cooperation with a German clinic specialized in bone surgery and endoprothetic, the presented scenario describes a standard procedure for hip replacements. In this process, the patient usually moves back and forth between different physicians/specialists and a clinic to receive an in-depth diagnosis as well as appropriate medical care and treatment. A patient typically starts with consulting a family doctor, is directed to a specialist, chooses a hospital, goes through consultation and registration at the hospital with a schedule for further preparation, passes through all preparations, stays in the hospital where the operation is performed, followed by aftercare treatment at specialists, (see figure 1). In the course of this process, various documents have to be exchanged, some of which are delivered by the patient while others are sent by mail or fax.

\subsection{Problems and Different Perspectives}

The performance of this sample process is affected by a number of problems inherent in the nature of health service in general: 
- Process Responsibility. There is no overall responsibility for the process or its planning. Rather, the process seems to rely on the ability of individual providers to shape the service according to their special insights about the patient's case including the calling in of additional providers.

- Monitoring Process Status. Involved providers fall short of a complete picture of the process, its current status and development. Often, they lack information about deviations from tacitly assumed ways to proceed, or are unable to retrace this knowledge.

- Timely Exchange of Documents. A further problem relates to the exchange of documents. Today, there are no clear rules. It is neither obvious in which way documents are to be delivered nor is it fixed which kind of documents should be exchanged at all.

Apart from these problems inherent to the process, a further set of obstructions is caused by the patient's multiple crossing of two different "realms" of funding (different procedures for reimbursing physicians and hospitals). While trying to enforce more efficient cooperation among the "separated" providers the new reimbursement laws seem to fall short of providing the necessary incentive for the resulting increasing coordination. Considering the different perspectives of the two provider groups makes this clear.

- Specialist's perspective. Without any kind of extra reimbursement, the specialist might be unwilling/uninterested to spend time on improving the hospital's knowledge about the patient's overall condition more than absolutely necessary. On the other hand, the specialist is eager for information about the patient's treatment in the hospital or at least at the time of discharge so that he/she is able to attend the patient in a circumspect and professional manner.

- Hospital's perspective. According to the new reimbursement laws, where the patients overall condition affects payment (e.g. a hip replacement for a patient with a heart condition will be more expensive than for an otherwise healthy patient), the hospital needs to receive this basic and from a medical perspective anyhow desirable information. Furthermore, the capacity utilization of the operating theatre forms a critical profit factor. For this reason, the hospital is highly interested in the patient's status of preparation prior to admission. If a lack of preparation is discovered only then, the probability of the operating theatre staying idle the next day is high.

\subsection{General Requirements for Service Delivery in Health Provider Networks}

The example makes it clear that a better support of cross-organizational health service processes is needed. However, the requirements are far from trivial. As we have seen, standard procedures including the exchange of an increasing amount of 
information have to be worked out, agreed upon and supported. At the same time, flexibility is required in order to enable deviations from standard procedures. Different kinds of overviews (over the process status of one patient, of all patients of a certain provider in a certain status, etc.) have to be provided.

These sophisticated requirements on process performance clash with missing standards in software and hardware equipment and sometimes poor motivation to use computers still perceptible among health providers. Resulting technical platforms had better not require any installation and maintenance work at providers' sites. The main focus of physicians respecting (caring about the patient), the provided systems should be as easy to use as possible. In addition, availability, stability and security are priorities.

\section{XML-Based Serviceflow Management}

With serviceflow management (SFM), we introduce a general concept which was developed for supporting interrelated personalized and situated services carried out across different organizational units or provider firms. It is based on the notion of serviceflow and provides a modeling approach together with a generic XMLbased architecture.

Above all, SFM is based on services understood as relationships, since this is more comprehensive than just focussing on services as encounters [Gute95]: Services are social relations to recognize and satisfy situated needs of an individual or collective actor, based on an explicit or implicit agreement. ${ }^{l}$ It is often a matter of trust whether the client calls for a specific service, and in the end it is only the client who decides about successful satisfaction of his needs ${ }^{2}$. One of the challenges of introducing computer support in the service domain lies in this situated and personalized relation between service provider and client [Klis00].

However, this personal dimension has to be enhanced by a dimension of professional performance. From the perspective of the professional provider, service must be defined in economic terms [KIWe00]: A service is an act of labor or a performance by a business organization, the net value of which is based on the recognition and satisfaction of customer needs. To this end, standard processes are, where possible, adapted to the requirements of the individual service situation.

1 Emphasizing this perspective matches perfectly with the healthcare environment, where trust in and loyalty to the doctor are often long lasting.

2 Special difficulties observed in assessing health services such as the delay between the treatment and cure or the patient's inability to assess the skills of a physician are pointed out in [GaHo98]. Nevertheless, dissatisfaction is a general observed reason causing patients to change their service provider. 
With the notion of serviceflow we apply this twofold understanding of services their relationship and efficiency aspects - to interrelated services which consist of sequences of sub-services which are delivered by different service providers. The success of these services (in terms of business revenue) is crucially dependent on the customer's/client's experiencing all sub-services as coherent and continuous and as part of a comprehensive overall serviceflow. From the customer's perspective, a serviceflow gives customers the feeling of being embedded in a coherent "flow of services" taken care of by the service. From the service provider's perspective, the emphasis is on the integration and coherence of all situated sub-services across temporal, spatial and team boundaries.

To simplify matters, we define serviceflow in terms of servicepoints. A service always creates some social situation, it needs "places" [HaDo96] which frame the situation where service tasks are carried out. These places we call servicepoints, and the successive interrelation of a number of servicepoints is a serviceflow.

Focussing on service providers, the challenge is to look for recurrent serviceflow patterns. For defining these patterns, both the sequence of servicepoints and the service at each servicepoint have to be modeled. The sequence of servicepoints for our example is shown in figure 2 . It forms an entry point to our web-based modeling space. Each servicepoint captures specific service tasks to be carried out and their respective pre- and postconditions from the provider's point of view. The pre- and postconditions represent the contract for interrelating the servicepoints. Service tasks are modeled as UML use cases with each use case being further linked to a rich description (a scenarios and use case picture). Cooperation pictures can augment the serviceflow representation to further illustrate cooperation among the involved actors, for more detail see [Kli*01].

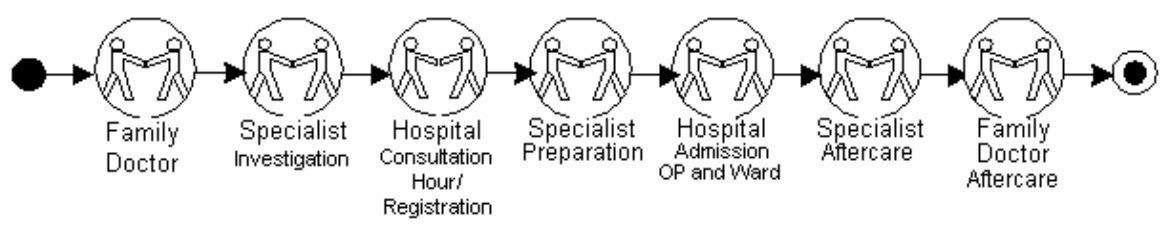

Figure 2: Model of the Case Serviceflow

Serviceflow management is now centered around the technical representations of the modeled process pattern that lead to the notion of servicefloat and servicepointscript. Servicefloats are sent from servicepoint to servicepoint and capture personalized, always up-to-date process knowledge, whereas servicepointscripts direct the standard activities at each servicepoint (see figure 3). This approach bears the following obligations and potentials: 


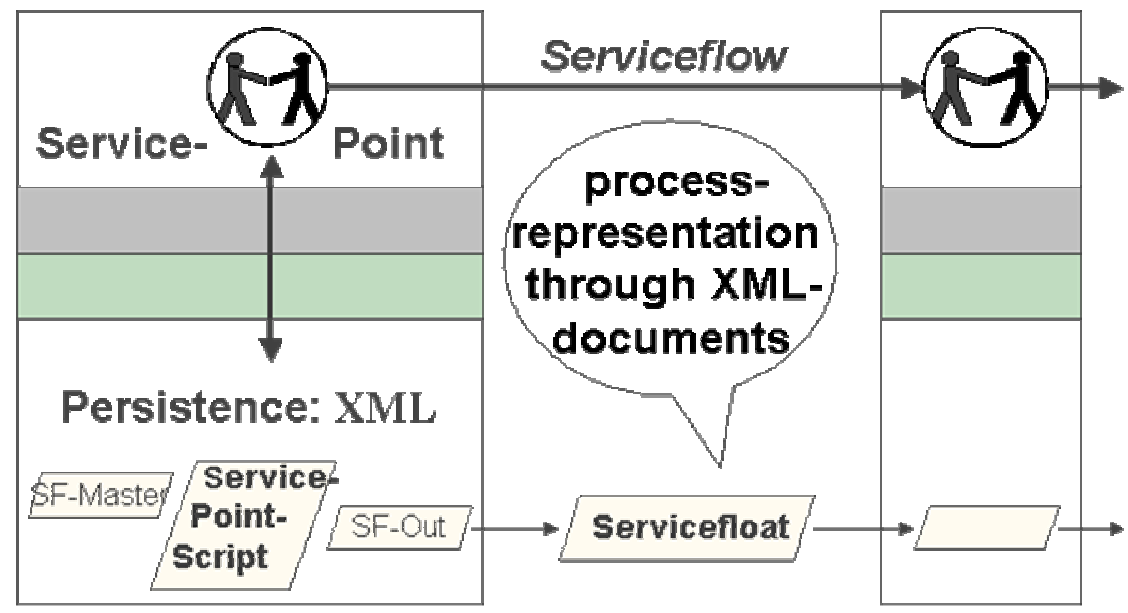

Figure 3: Servicefloats and Servicepointscripts

- Initializing a servicefloat by copying (and possibly adapting) a standard serviceflow pattern guides each provider as to how to deliver the service.

- Enabling providers to access and update the process representations (as a material) allows for flexibility and instant realization of changes.

- Documenting the history enables a service provider to be informed about deviations from the standard and their reasons.

- Constant update of the current and next servicepoints forms a basis for automating the delivery of servicefloats to the next provider.

All in all, SFM requires agreements on the content of the modeled serviceflow pattern and on the handling of these representations during exchange.

In order to exchange servicefloats between provider organizations, we represent them technically as XML documents. As the use of XML is only just spreading [LeOb01] and only a few domain specific solutions are available as yet, we had to develop our own framework for an XML-based process representation for serviceflow management.

The framework requires network members to agree upon a set of XML-DTD for servicefloats and servicepointscripts and other shared data structures (e.g. forms, patient data, patient record), XML "master"-documents for servicefloats and servicepointscripts according to different serviceflow types, and a set of rules on how to manipulate and share those XML documents.

In detail, a servicefloat's XML representation contains the following elements: an identifier for individual serviceflow, basic information on the serviceflow client, the current servicepoint (servicepoints are described by identifier, name, type, 
provider, address), a list of scheduled servicepoints, a list of servicepoints passed, a list of accumulated postconditions, a list of documents, i.e. short message texts or references to full documents or document folders. At each servicepoint, the servicefloat is evaluated according to the respective servicepointscript that prescribes the activities at the 'current servicepoint': an identifier for the individual servicepoint, basic information on the servicepoint provider, current activity (activities are described by identifier, name, type, task), a list of scheduled activities, a list of passed activities, a list of preconditions for the set of activities at this servicepoint and a list of documents.

\section{Networking Healthcare Providers}

This approach to serviceflow management does not presuppose any kind of IT infrastructure except the processing and exchange of XML documents. Thus, any provider can easily join the cooperative serviceflow management and may independently care for its own IT support as long as it keeps up with the mutual agreement. However, we are interested in a generic layered architecture supporting servicepoints at providers' sites, which will be introduced below. On this basis, we discuss contrary aspects underlying possible technical network infrastructures for health providers and, finally, present a prototype to support our case example.

\subsection{Servicepoint Architecture}

There is no need to subscribe to a specific IT architecture to implement XML document handling at specific servicepoints. However, we recommend a clientserver architecture with three server layers:

- Frontend: client to present the user interface

- Interaction: server layer to organize the user dialogue

- Serviceflow application: server layer to realize the XML document processing for process representation

- Persistence: the server's file system or data base for saving and retrieving XML documents

The IT architecture for a web-based servicepoint as applied in our e-health prototype is shown in figure 4. The user dialogue is organized on the basis of Java Server Pages. Alternatively, a set of templates created in a web content management system could be used. The user dialogue implementation includes Java method calls addressing the public interface of the serviceflow application layer implemented in Java. 
The components of the serviceflow application layer ${ }^{3}$ encapsulate the processing of the XML documents related to serviceflow management:

- the servicepoint manager includes methods for retrieving the relevant XML files, creating Document Object Models (DOM) of servicefloat and servicepointscript for a specific customer, saving the manipulated DOMs in XML files and preparing the servicefloat for dispatch

- servicefloat and servicepointscript both include a variety of get- and set-methods (according to the usage of serviceflow process representation) to be called on through the public interface for manipulating the respective XML DOM.

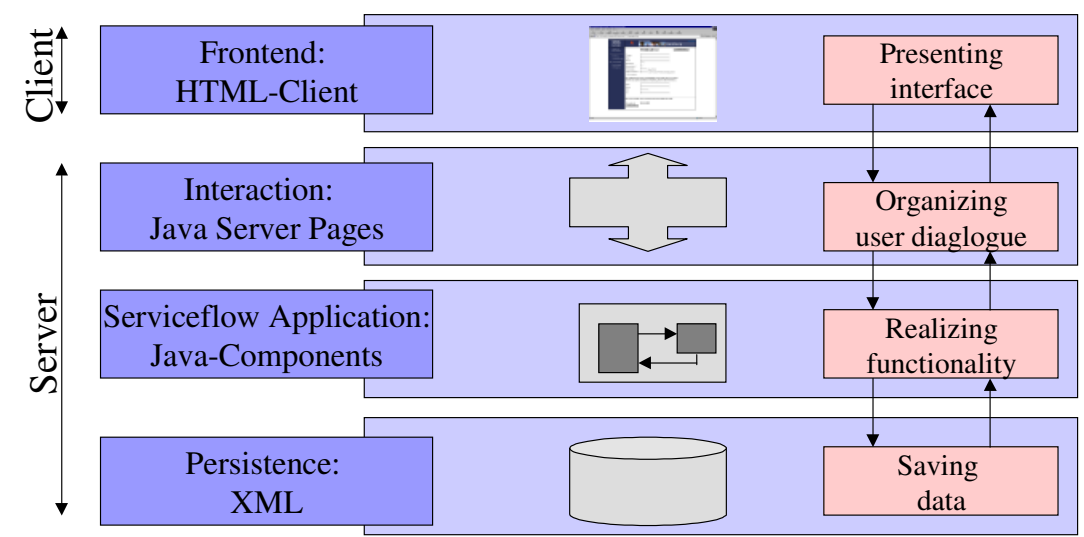

Figure 4: IT Architecture for a Web-Based Servicepoint

The described architecture is used in the current prototypical implementation for e-health services presented in the next section. In parallel, we are applying the same architecture within a project in order to realize an e-Government process portal, where the first service to be offered is the postal vote application in the city state of Hamburg through www.hamburg.de. The forthcoming results [KlWe01a; $\mathrm{K} 1 \mathrm{We} 01 \mathrm{~b}]$ will certainly contribute to the set of ready-made IT solutions to support serviceflow management in general, indicating how future e-heath service processes could be supported similarly or which kind of modifications are necessary.

\subsection{Information Flow vs Information System}

Returning to the general requirements for health service discussed in section 2, we note two contrary aspects in regard to suitable infrastructures in this domain. Inter-

\footnotetext{
${ }^{3}$ The description is based on the prototype developed at Hamburg University as presented at the end of January 2001.
} 
related services are delivered by sending the patient from one servicepoint to the other. At each servicepoint specific tasks are performed and documents exchanged. Flexibility can be achieved through the exchange of updated process knowledge along the service chain. Thus, the information flow seems to accompany the patient's move through the service provider network. However, depending on the kind of examination performed, service work might last longer than the patient's presence at a servicepoint. Accordingly, providers have to be able to add documents to a patient's case although the servicefloat has already been left the servicepoint. Furthermore, as patients often revisit the same provider during a serviceflow (though at different servicepoints) providers try to be kept informed about the patient's course at other sites throughout the whole serviceflow period.

It seems that the nature of interrelated health services requires a balanced solution somewhat in-between an information flow and information systems approach. Whereas the information flow approach emphasizes flexibility and autonomy without assuming any central technical infrastructure, the information systems approach highlights provision of information at any time for any provider while requiring some sort of central solution. Additionally, we have to consider the existing IT infrastructure in the health sector ranging from not seldom "poorly" IT equipped physicians to large hospitals using sophisticated IT landscapes including web servers. So far, different alternatives are on the horizon and will be discussed in the next subsection.

\subsection{The Prototype}

For our prototype we combined both directions discussed. We assume a central server for serviceflow management, i.e. servicefloats and servicepointscripts are used according to the rules but managed centrally instead of being exchanged between providers. The huge advantage lies in the resulting low requirements on technical and software equipment: Providers will need nothing more than an internet browser which matches with the requirements stated in section 2 .

Nevertheless, more sophisticated scenarios are on the horizon. Structurally, the patient record has to be separated from the process knowledge. Infrastructurally, an appropriate distribution of servers across a network of commercial and healthcare technical providers with different responsibilities has to be worked.

The web-based user interface at a servicepoint provides access to the underlying process information as well as to exchanged data. It has four main sections, as indicated in figure 5. Section 1 represents the provider/network leaving space for its corporate identity. Section 2 visualizes the actual serviceflow for the patient in question. Section 3 provides the list of activities to be carried out at a chosen servicepoint. Section 4 captures the patient record represented as a folder including forms and documents about the patient's treatment in the SF. When selecting a 
servicepoint rather than a patient's case, this area presents an overview over all patients served at this servicepoint.

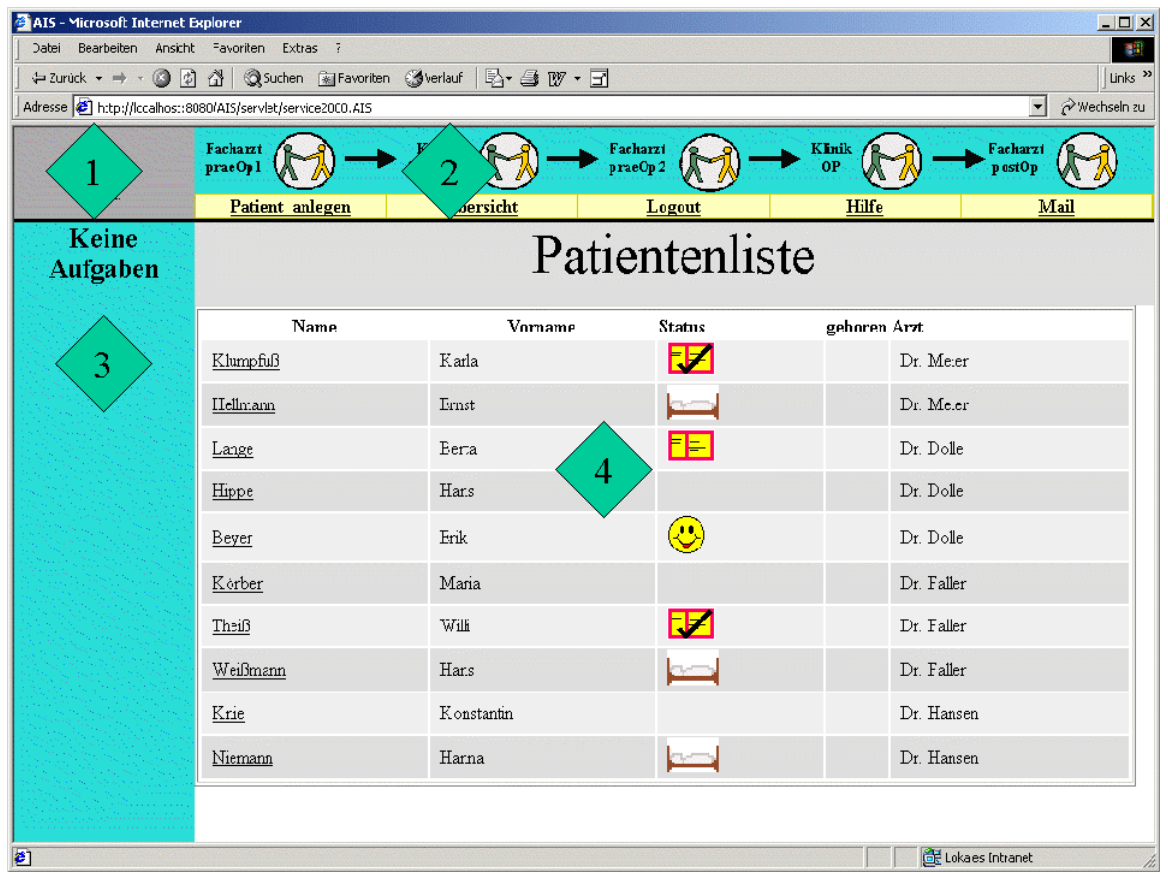

Figure 5: Overview over Patients

A typical work scenario could be as follows. A specialist starts the system and chooses the serviceflow "Preparation, performance and aftercare of an inpatient surgical operation". A window then opens with the serviceflow in question and an overview over his/her patients active in the serviceflow, out of which the specialist chooses a certain patient. As soon as this happens, the patient record with details about the patient's case appears, see figure 6. Depending on the point of service the patient is in, a task list with standard tasks at this point is provided. Clicking on tasks will open the patient's record at the right place, when tasks are accomplished they are automatically marked (or by hand). Even though possible in the underlying serviceflow representation and implementation, the interface still lacks supporting changes in the task list or in providing pre- and postconditions.

As a modification of the original SFM concept and owing to the "one server solution", the prototype permits information retrieval at any time about the patient's actual case as well as addition of documents to the process, which requires further change indicators (awareness information) at the overview and patient record level. 


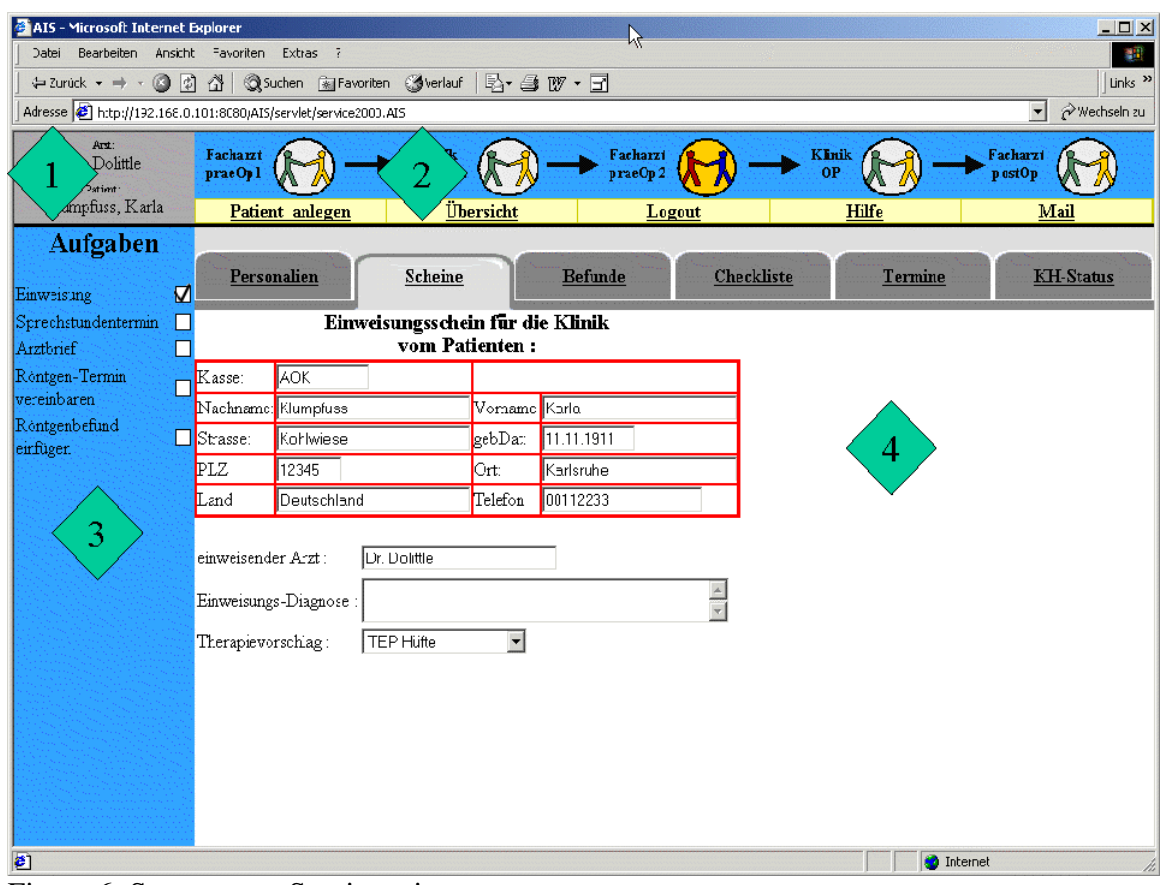

Figure 6: Support at a Servicepoint

\section{Summary}

Typical service processes in healthcare allow and force the patient to move between different specialized service providers. The patient has the chance to receive optimal comprehensive therapy and care, provided the necessary cooperation and coordination among the service providers works.

With serviceflow management the paper presents an approach that claims to be suitable for supporting flexible interrelated services as required in healthcare. SFM is oriented around services understood as relations between provider and customer and defines interrelated services in terms of chains of servicepoints. As pointed out, the exchange of servicefloats representing individualized current process knowledge enables situated changes. Furthermore, by cutting up processes into manageable parts (serviceflow and servicepoints), the modeling features provided nurture the necessary agreement process among network partners.

A realization is presented using XML representations of process knowledge based on XML DTDs for servicefloats and servicepointscripts. Additionally, domain specific XML standards for exchange of patient information can be integrated if existent. Along with these concepts and realization basics, we provide Java-based 
serviceflow management components which can be used at a provider's site if wanted.

So far the general serviceflow management concept. However, as we have seen, the healthcare sector needs a still more comprehensive solution. Three main reasons have to be considered. First, a healthcare serviceflow requires from some providers to be in charge over a longer period of time than the time frame of a single servicepoint would allow. Second, the exchanged diagnoses-related data are sophisticated and of a possible high data volume. Third, the IT infrastructure in healthcare is not too promising at the moment. Consequently, a combination of both an information flow and information systems approach seems appropriate. With our prototype for the considered case we provide a first solution which combines serviceflow management flexibility using XML based process representations and generic Java-components with a one-site server solution providing access to patient records at each point in time. For the future, we expect infrastructures based on a mixture of commercial application service providers and selected healthcare providers for equipping healthcare networks with appropriate IT-support.

\section{Acknowledgements}

The authors thank the Lubinus Clinic in Kiel and further interview partners from practices for the opportunity to analyze the case presented and for sharing many interesting aspects concerning health provider networks. Furthermore, we wish to thank the student members of our hospital project, Lawrence Cabac, Heike Hager, Anja Hennemuth, Sylvia Oelkers and Sang-Il Kim, and Timmy Blank and Nol Shala for implementing the DTDs and generic Java components.

\section{References}

[Ande97] Anderson, J.G.: "Clearing the Way for Physicians' Use of Clinical Information Systems", Communications of the ACM, Vol. 40, No. 8, 1997, pp. 83-90.

[GaHo98] Gabbott, M. and G. Hogg: Consumers and Services, John Wiley\&Sons, 1998.

[Gute95] Gutek, B.: The Dynamics of Service, San Francisco: Jossey-Bass Publishers, 1995.

[HaDo96] Harrison, S., Dourish, P.: Re-Place-ing Space: The Roles of Place and Space in Collaborative Systems, Proceedings CSCW'96, pp. 67-76.

[Klis00] Klischewski, R., Abstrakte Bedürfnisse und konkrete Beziehungen - oder: Wie man Services (nicht) modelliert, Proceedings Modellierung 2000, pp. 19-26. 
[K1We00] Klischewski, R., Wetzel, I., Serviceflow Management, Informatik Spektrum, vol. 23 (1), February 2000, pp. 38-46.

[Kli*01] Klischewski, R., Wetzel, I., Bahrami, A.: Modeling Serviceflow. In print: Proc. of the $1^{\text {st }}$ International Conference on Information Systems Technology and its Applications (ISTA). Kharkiv, Ukraine, June, 2001.

[KlWe01a] Klischewski, R., Wetzel, I.: XML-based Process Representation for E-Government Serviceflows. To appear in Conf. Proc. of the $1^{\text {st }}$ IFIP Conference on E-Commerce, E-Business, E-Government, Zürich, Oct. 2001.

[KlWe01b] Klischewski, R., Wetzel, I.: Serviceflow Management für organisationübergreifendes eGovernment. To appear in Proc. of Informatik 2001, (Jahrestagung GI/ÖCG), Workshop Electronic Government, Wien, Sept. 2001.

[Kra*96] Krabbel, A., Wetzel, I., Ratuski, S.: Participation of Heterogeneous User Groups: Providing an Integrated Hospital Information System, In: Proceedings of the Participatory Design Conference (PDC'96), Cambridge, Massachusetts, USA, pp. 241 - 250, 1996.

[KrWe00] Krabbel, A., Wetzel, I.: Designing Hospital Information Systems: Handling Com-plexity via a User-Oriented Document-Based Approach, in: A. Armoni (ed): Healthcare Information Systems: Challenges of the New Millennium, Idea Group Publishing, pp 1-26, 2000.

[LeOb01] Lenz, K., Oberweis, A.: Modeling Interorganizational Workflows with XML Nets. Proceedings of the 34th Annual Hawaii International Conference on System Sciences (HICSS-34). IEEE, 2001

[Mint79] Mintzberg, H.: The Structuring of Organizations, Prentice Hall, 1979.

[Ripp00] Rippen, H.: e-Health Code of Ethics, , Journal of Medical Internet Research 2000,; 2 (2): e9, URL: http://www.jmir.org/2000/2/e9/

[Rodr00] Rodrigues, RJ.: Ethical and Legal Issues in Interactive Health Communicatinos: A Call for International Cooperation, Journal of Medical Internet Research 2000,; 2 (1): e8, URL: http://www.jmir.org/2000/1/e

[Str*97] Strauss, A. L., Fagerhaugh, S. Suczek, B. Wiener, C.: Social Organization of Medical Work, Transaction Publishers, 1997.

[Tam*00] Tambouris, E., Williams, M.H., Makropoulos, C.: Co-operative Health Information Networks in Europe: Experiences from Greece and Scotland, Journal of Medical Internet Research 2000,; 2 (2): e11, URL: http://www.jmir.org/2000/2/e11/

[WoKa97] Wolf, C.G., Karat, J.: Capturing What is Needed in Multi-User System Design: Observations from the Design of Three Healthcare Systems, Proc. of DIS, Amsterdam, 1997.

[Wetz01] Wetzel, I.: Information Systems Development with Anticipation of Change: Focussing on Professional Bureaucracies, Proc. of the Hawai'i Int. Conf. on System Sciences, HICSS-34, Maui, 2001. 